\title{
Protée
}

\section{Le recadrage dans la naissance des idées innovantes ou comment favoriser la créativité en s'inspirant des théories développées par les SIC}

\section{Céline Bryon-Portet}

Volume 39, numéro 1, printemps 2011

URI : https://id.erudit.org/iderudit/1006731ar

DOI : https://doi.org/10.7202/1006731ar

Aller au sommaire du numéro

Éditeur(s)

Département des arts et lettres - Université du Québec à Chicoutimi

ISSN

0300-3523 (imprimé)

1708-2307 (numérique)

Découvrir la revue

Citer cet article

Bryon-Portet, C. (2011). Le recadrage dans la naissance des idées innovantes ou comment favoriser la créativité en s'inspirant des théories développées par les SIC. Protée, 39(1), 103-110. https://doi.org/10.7202/1006731ar

\section{Résumé de l'article}

Existe-t-il des outils contribuant efficacement à améliorer le processus d'innovation, et, si oui, les Sciences de l'information et de la communication (SIC) peuvent-elles participer de ce processus ? Nos recherches nous ont amenée à explorer une abondante littérature scientifique, puis à nous entretenir avec des enseignants chercheurs spécialisés dans le génie chimique et le génie industriel, secteurs particulièrement tournés vers l'innovation. Nous avons alors décelé des similitudes entre certaines méthodes destinées à stimuler la créativité, et des notions familières aux théoriciens de la communication. Nous avons pu formuler l'hypothèse que de nombreuses pratiques élaborées dans le domaine de l'ingénierie et du management de projet, visant à impulser l'innovation, utilisent le concept de recadrage tel que les chercheurs du Collège invisible l'ont défini et développé, sans pour autant le nommer comme tel ni même le formaliser. Nous en ferons ici la démonstration à partir de l'étude de quelques exemples concrets, et en nous appuyant sur les théories fondatrices de la pensée innovante élaborées par Genrich Altshuller et Edward de Bono. 


\title{
LE RECADRAGE DANS LA NAISSANCE DES IDÉES INNOVANTES OU COMMENT FAVORISER LA CRÉATIVITÉ EN S'INSPIRANT DES THÉORIES DÉVELOPPÉES PAR LES SIC ${ }^{1}$
}

\author{
CÉlINE BRYON-PORTET
}

Les concepts de cadrage et de recadrage dans le processus de changement psychologique et de créativité

Des concepts majeurs du Collège invisible

L'un des apports majeurs du Collège invisible ${ }^{2}$ qui s' est mis en place à partir des années 1950 réside dans le paradigme systémique que ses chercheurs ont développé, poursuivant en cela la voie ouverte par le père de la cybernétique, Norbert Wiener, tout en I'approfondissant et en la modifiant sensiblement. L'interdisciplinarité de ce réseau de chercheurs, informel mais fondateur, explique en partie la démarche globale qui fut celle de ses membres. Issus de domaines aussi variés que I'anthropologie, la sociologie, la linguistique ou encore la psychiatrie, ces derniers ne pouvaient qu'être sensibles à une approche totalisante des questions posées dans le champ des SIC. L'enrichissement et l'influence réciproques des chercheurs favorisaient bien évidemment un décloisonnement des savoirs et la prise en considération de paramètres divers, relevant de domaines variés.

Ainsi, par rapport au modèle dit «télégraphique » de Claude Shannon et de Warren Weaver, quelque peu réducteur puisqu'il tendait à privilégier une conception mathématique, linéaire et informationnelle, le Collège invisible introduisit une vision élargie de la communication, où le contexte global revêtait une importance toute particulière, au moins aussi cruciale que le fait même de l'énonciation. Dans cette perspective, Gregory Bateson et Ray Birdwhistell, notamment, jouèrent un rôle pionnier. Le second, par exemple, forgea l'idée d'une «communication intégrative» qui envisage toute action de communication dans un contexte plus vaste que le simple contexte interactionnel. Ce chercheur alla jusqu'à englober des données, telles que l'organisation de l'espace (architecture, décoration intérieure, etc.), dans la mesure où celle-ci est le fruit d'un agencement environnemental conscient et volontaire de la part de l'homme, et par conséquent porteur de sens pour celui qui sait déchiffrer ces signes. Paul Watzlawick, lui aussi, étendit le concept de communication. Il mit en avant la relation, parallèlement à l'information, et montra que ces deux éléments agissent respectivement en tant que forme et en tant que contenu, représentant deux aspects interdépendants de toute situation de communication.

Grâce aux recherches menées par l'École de Palo Alto, et plus précisément du MRI (Mental Research Institute), la dimension situationnelle de la communication (communication conçue comme un système dont chaque partie est liée au tout et est susceptible d'avoir un impact sur l'évolution générale, en créant un phénomène de rétroaction) est apparue comme primordiale. C'est dans cette optique situationnelle que la notion de «cadre» ou de «cadrage» doit se comprendre. Le cadre peut être défini comme le contexte de référence global dans lequel s'inscrit une action de communication. Les interactions des acteurs en présence, les codes sociaux utilisés, l'environnement spécifique dans lequel se déroule un événement forment une sorte de trame et donnent lieu à des représentations, des comportements et des interprétations. Ainsi les échanges interpersonnels ne prennentils de sens que relativement au cadre qui est le leur.

Erving Goffman s'est également penché sur l'analyse de ces «cadres» au sein desquels se déroule toute expérience, et qui peuvent se superposer à d'autres cadres, créant alors différents degrés de cadrage. Dans son ouvrage intitulé Les Cadres de 
l'expérience (1974), il distingue ainsi entre «cadres primaires» («naturels » ou «sociaux») et «cadres transformés» (par «modalisation» ou par «fabrication»). Les cadres transformés par modalisation, c'est-à-dire par un processus de transcription qui change l'interprétation d'une situation, ou plutôt du sens que celle-ci avait dans un cadre primaire, sont ceux auxquels nous allons nous intéresser au cours de cette étude. Notons que le terme même de «cadrage " n'est pas sans évoquer un registre de langue inhérent à la photographique et aux productions cinématographiques. II indique clairement que les schèmes auxquels nous faisions précédemment allusion équivalent à des angles de vue particuliers, dont la nature a des implications sur la perception que l'on peut avoir d'une scène, d'un sujet ou d'un objet. Quant à la notion de recadrage, elle n'est autre que le passage d'un cadrage à un autre cadrage, d'un modèle perceptif à un autre modèle.

Les opérations de recadrage dans le domaine thérapeutique: problématique du changement et approches constructivistes

Le recadrage est donc étroitement lié -directement ou indirectement - à la problématique du changement (Watzlawick et alii, 1975). L'efficacité des opérations de cadrage et de recadrage a été prouvée, notamment dans le domaine thérapeutique, largement balisé par l'École de Palo Alto. Ainsi la «thérapie brève » consiste-t-elle à utiliser le recadrage (mais également "l'injonction paradoxale» liée à la notion de «double contrainte») afin de modifier la signification qu'une personne donne à une situation, c'est-à-dire à lui apporter un éclairage différent entraînant une redéfinition du sens du contexte dans lequel elle se trouve impliquée. En effet, selon Paul Watzlawick, tout symptôme doit être considéré comme une tentative d'adaptation à un contexte particulier. Cependant, cette tentative échoue, la plupart du temps, parce qu'elle s'appuie sur une vision erronée du contexte. Watzlawick souligne que nous faisons nous-mêmes notre malheur (1984), car nous reproduisons des solutions qui se sont avérées efficaces par le passé alors que le contexte, lui, n'est plus le même. D'où l'échec du malade à trouver la voie de la guérison sans l'aide d'un thérapeute capable de l'extraire de ce déterminisme situationnel et de le guider vers une approche originale du problème.

Le traitement des maladies mentales - et plus particulièrement de la schizophrénie - élaboré par le MRI repose sur une vision interactionnelle, constructiviste et systémique. En effet, cette dernière postule que le processus de changement s'appuie sur la réorganisation d'éléments en interrelation et la reconstruction des représentations que nous nous forgeons de la réalité. Les prémisses théoriques de ce mode de traitement clinique sont la non-séparation du sujet et de son environnement, du patient et de la représentation qu'il a de son problème. $C^{\prime}$ est en modifiant radicalement leurs constructions mentales, les modèles qui déterminent le déchiffrage quotidien de leur environnement, que les individus peuvent adopter une nouvelle façon d'être. De telles prémisses, qui s'opposent à une conception monadique de l'individu, sont communes à toutes les approches constructivistes. En effet,

[...] pour le constructivisme, toute connaissance est une construction du sujet. Le sujet se réfère à des finalités lorsqu'il construit son savoir. Ainsi la dualité sujet/objet est-elle dépassée et, dans ce cadre, de l'interaction entre les deux termes émerge et se construit la connaissance - la connaissance est une action.

(Russo, Rea et Salamanca-Avila, 2004 : 175)

Alex Mucchielli, spécialiste des approches constructivistes, résume parfaitement cette approche, en précisant ce que recouvre le procédé de recadrage chez Paul Watzlawick:

[...] recadrer, pour Watzlawick, c'est donc redéfinir la situation ou passer à une méta-vue de la situation dans le but de changer le sens des rapports entre les acteurs. La notion de "recadrage» est ainsi fondamentale pour toutes les études sur le changement de comportement. Pour modifier une conduite il s'agit essentiellement de modifier le système dans lequel la conduite est mise en œuvre, car, dans le contexte modifié, la conduite en question prend un autre sens qui n'apparaît plus alors comme pertinent à l'acteur. (2004: 17)

Or, ces notions de cadrage/recadrage, étudiées par l'École de Palo Alto, tant au plan théorique qu'au plan expérimental, possèdent des applications plus larges que celles relatives aux SIC sticto sensu. Si I'application du recadrage à des fins thérapeutiques est connue pour avoir été diffusée par Gregory Bateson et Paul Watzlawick, entre autres, il existe d'autres utilisations possibles de ce procédé, qui peuvent se révéler également prometteuses. Avant de montrer que la méthode du recadrage se révèle particulièrement profitable dans tout processus inventif, nous allons nous efforcer de prouver qu'il s'avère un précieux outil de créativité.

\section{La remodélisation comme outil de créativité}

dans les sciences et les arts

En tant que référent global, tout cadre participe d'un certain conditionnement. De lui dépendent en partie nos représenta- 
tions symboliques, notre interprétation d'une situation. Il est comme le creuset ou le prisme à travers lequel le sujet observant appréhende un fait. Dans une certaine mesure, un cadre agit comme une grille de lecture apposée sur ce que l'on nomme «la réalité». Il influence le mode de saisie, de réflexion, et, partant, le comportement humain. Or, si l'on admet qu'innover consiste à considérer un objet donné (procédé, produit ou service) d'une manière différente, c'est-à-dire avec un regard "neuf», force est de reconnaître que le cadre joue un rôle de premier ordre puisqu'il peut constituer un obstacle (ou au contraire un facteur clé de succès) dans cette démarche, ainsi que nous le montrerons à travers quelques exemples concrets. C'est la raison pour laquelle le cadrage, en tant que remodélisation, peut servir à modifier un cadre inadéquat et à créer simultanément des conditions optimales pour faire émerger une pensée originale.

L'une des illustrations les plus anciennes de cette méthode de transfert nous est donnée par la culture grecque. Au plan philosophique, la célèbre allégorie de la caverne exposée par Platon au livre VII de La République est un exemple significatif de recadrage. Lorsqu'il est enchaîné dans les profondeurs ténébreuses, le prisonnier perçoit des ombres, qu'il prend pour des objets, alors qu'elles n'en sont que le reflet déformé. Mais lorsqu'il redescend, après avoir été libéré et avoir contemplé la lumière du soleil, symbole de la connaissance, le philosophe ne perçoit plus les ombres qui se meuvent comme des objets réels. Il a conscience de leur véritable nature et les considère comme de vulgaires copies, car son regard, au lieu d'être dirigé vers les parois trompeuses de la caverne, est fixé sur les montreurs de marionnettes, de l'autre côté. Son point de vue n'est plus le même. À l'inverse, les autres prisonniers continuent d'avoir une vision tronquée. Entre le philosophe et ces derniers, s'interpose un cadrage différent. Chez le prisonnier libéré, en effet, est intervenu un recadrage de la situation, une modification de la vision, qui l'éloigne irrémédiablement de la vision de ses confrères. Deux systèmes de croyances, deux systèmes de représentation, s'opposent alors. Cependant, I'analogie entre la conception platonicienne du changement et celle établie par les chercheurs du MRI s'arrête là. Car si les seconds possèdent une conception constructiviste, et partiellement relativiste, de la réalité, Platon, quant à lui, affirme la supériorité d'un monde idéel, par rapport au monde sensible qui n'en serait qu'une copie dégradée ou, pour le dire en termes kantiens, la prééminence du monde nouménal sur le monde phénoménal. Cet idéalisme, on en conviendra, se trouve aux antipodes de tout constructivisme radical.
Au plan scientifique, une seconde illustration de la méthode de recadrage nous est donnée par Thalès. Lorsque ce dernier entreprend de mesurer la hauteur de la pyramide de Khéops, il se détourne, assez singulièrement, de l'imposante construction qui se trouve face à lui, préférant prendre l'ombre pour référent plutôt que l'objet lui-même. Ce faisant, il change de cadre de référence, déplaçant son champ d'investigation afin de résoudre les problèmes théoriques et pratiques qui se posent à lui. Ce détour, qui est aussi un détournement, contient en lui - au moins en germe - toute la philosophie qui donnera plus tard naissance aux techniques de recadrage. Paraphrasant Michel Serres, Michel Callon va jusqu'à voir dans le théorème de Thalès une expérience inaugurale, symbole même de toute démarche scientifique, par la mise à distance objective à laquelle elle procède. Il prend d'ailleurs le contre-pied du proverbe affirmant qu' « est fou celui qui lâche la proie pour l'ombre », et déclare que la science lâche précisément la proie pour l'ombre, la pyramide matérialisée pour son reflet fugitif, projeté par le soleil sur le sol égyptien. Et de poursuivre en affirmant qu' « une expérience n'est jamais innocente. Son montage, son interprétation sont largement dépendants des présupposés théoriques des chercheurs qui la réalisent» (Callon, 1988: 9).

Les théories physiques qui ont vu le jour au XXe siècle semblent d'ailleurs corroborer les dires de Michel Callon. Expliquant les principes fondateurs de la physique quantique, Étienne Klein (1997) met ainsi en exergue le fait que la lumièrepeut être tour à tour considérée comme phénomène ondulatoire et comme phénomène corpusculaire, alors même que les ondes et les corps sont conçus comme exclusifs, irréductibles et contradictoires, selon une approche scientifique traditionnelle. Mais, plus qu'une dualité, cette expérience présentée par la physique quantique démontre l'importance primordiale du cadrage. Entre la physique classique, celle de Newton et de Copernic, et la physique de Planck, Heisenberg, Maxwell et Bohr, il y a une différence de cadrage, comme il y avait une différence de cadrage entre le philosophe libéré et les prisonniers enchaînés au fond de la caverne. En effet, l'expérience qui conclut au caractère ondulatoire de la lumière dépend du cadrage adopté par le sujet observant le phénomène, et des instruments de mesure que celui-ci utilise. L'apparente divergence de l'expérience qui exprime, quant à elle, la nature corpusculaire de la lumière est également due à la perspective expérimentale choisie. À l'aide $d^{\prime}$ 'une terminologie quelque peu différente, Thomas Kuhn ne dit pas autre chose dans La Structure des révolutions scientifiques. Les changements de paradigme qu'il recense dans l'histoire des 
sciences ne sont rien d'autre que des recadrages, des passages d'un système de référence à un autre.

Soucieux de ne pas réduire la connaissance à un relativisme absolu, tout en conservant les principes constructivistes de la méthode de recadrage, Paul Watzlawick (1977) propose de considérer qu'il existe "deux réalités ", l'une correspondant aux propriétés purement physiques des choses, l'autre ayant trait à l'attribution d'une signification à ces choses, aux valeurs socioculturelles sur lesquelles se fonde la communication. C'est ce second niveau, constitué de représentations, cette "réalité de la réalité » qui est susceptible, selon Paul Watzlawick, d'être modifiée par recadrage. Quelques années après la parution de son ouvrage How Real is Real?, Watzlawick approfondira cette «science paradoxale du changement » dans Les Cheveux du baron de Münchhausen (1991), où il élargira la perspective thérapeutique qui fut d'abord la sienne. Or, une étude attentive des méthodes d'innovation proposées par des chercheurs reconnus, dans le domaine de l'ingénierie et du management notamment, révèle précisément l'utilisation de ce procédé de déplacement du point de vue par transformation du cadre de l'analyse et de l'expérience. Largement utilisé, le procédé en question n'est pourtant pas souvent conceptualisé, ce qui nous paraît dommageable. En fait, il semble davantage relever d'une pratique intuitive que d'une pratique raisonnée, mais n'en apparaît pas moins crucial.

L'ouvrage d'Arthur Koestler intitulé Le Cri d'Archimède apporte à ce sujet des précisions extrêmement intéressantes, puisqu'il entreprend de démontrer que toute l'histoire des innovations scientifiques, mais également artistiques, repose sur un acte "bisociatif», soit la capacité d'associer des éléments logiquement incompatibles. Selon lui, l'esprit créatif, de quelque domaine que celui-ci relève, procède d'une aptitude à déplacer l'angle d'approche d'une chose ou d'une situation. Dans notre étude, nous avons pris le parti d'analyser principalement des exemples issus du domaine communicationnel (par le champ thérapeutique notamment), technique et «managérial ». Cependant, nous aurions également pu illustrer nos propos grâce à I'histoire de la littérature, de la musique ou de la peinture, par exemple. Que l'on songe à l'innovation que la peinture impressionniste, ou pointilliste, représente par rapport à la peinture classique de type réaliste, et au changement d'angle qu'elle implique (se tenir éloigné du tableau afin d'en saisir la cohérence globale au lieu de regarder la finesse et la précision des détails, etc.), et I'on comprendra qu'un même procédé de recadrage est chaque fois à l'œuvre. La révolution artistique que provoqua le mouvement surréaliste illustre peut-être mieux encore ce phénomène de conversion du regard présent dans toute innovation, et permet d'appréhender pleinement l'acte bisociatif tel qu'il a été défini par Arthur Koestler. Lorsqu'il écrit ses Manifestes du surréalisme, en 1924 puis en 1930, André Breton, en fin de compte, n'agit pas différemment du chercheur qui s'engage dans la voie innovante de la physique quantique. Le premier associe des mots et des images improbables (par le procédé de l'écriture automatique, entre autres), il cherche ce «point de l'esprit d'où la vie et la mort, le réel et l'imaginaire, le passé et le futur, le communicable et l'incommunicable, le haut et le bas cessent d'être perçus contradictoirement» (1985: 72-73); le second conjugue des représentations que la logique aristotélicienne et la physique traditionnelle jugent incompatibles (le chat de Schrödinger est à la fois mort et vif, la lumière peut être considérée comme corpusculaire et ondulatoire, etc.).

Le traitement de la question urbaine qui fut au cœur des pratiques littéraires et artistiques du mouvement situationniste pourrait également venir étayer nos propos. Lorsque les situationnistes entreprirent de reconsidérer l'architecture, ils voulurent en faire un instrument de rêve, de connaissance et d'action, lui donnèrent un rôle ludique et une forme modulable, mais surtout, ils lui reconnurent la capacité de créer des ambiances inédites et de réinventer la vie. Désireux d'insuffler une dimension poétique à la ville, ils entendirent lutter contre une fonction purement utilitaire de l'habitat, un « réductionnisme fonctionnaliste» des monuments, et en faire des lieux riches en surprises (Simay, 2008). La réorganisation de l'espace urbain (jugé trop rationnel depuis les grandes percées haussmanniennes), qui était censée redonner sa plasticité à la ville et soutenir la réinvention du quotidien, exigeait des qualités créatives. Les idées inventives qui devaient soutenir cette révolution urbaine, les situationnistes allèrent les chercher, la plupart du temps, en mettant en place des expériences susceptibles d'opérer des recadrages: après avoir préconisé la pratique de "la dérive», et élaboré toutes sortes de dispositifs de désorientation défamiliarisante prenant le labyrinthe pour modèle, un Guy Debord détourna les cartes des villes en les superposant, favorisant ainsi des connexions inattendues et reconstituant des territoires nouveaux.

Certes, ces différents exemples témoignent du fait qu'il existe différents types de recadrage et de degrés dans ce procédé, allant d'un simple changement de perspective adopté sur un objet ou une situation, à un déplacement de la pensée, parfois aléatoire, vers un cadre tout à fait différent en vue de perturber les processus de la connaissance. Mais, chaque fois, il s'agit de convertir 
le regard en modifiant un cadre de référence afin de dépasser les contraintes d'un dualisme logique, d'un rationalisme étriqué ou plus simplement de structures mentales trop rigides. Arthur Koestler a parfaitement résumé cet impératif en citant une phrase de Souriau: "pour inventer, il faut penser à côté » (Koestler, 1965 : 128). Cependant, la difficulté semble résider dans le fait de parvenir à systématiser cette pensée de l'à-côté, à trouver une méthode susceptible d'augmenter les chances d'inventivité au lieu d'en faire le fruit du hasard. Tel est précisément le défi que se sont lancé Genrich Altshuller et Edward de Bono, dont nous allons tenter de saisir les points de convergence.

\section{Méthodes de recadrage favorisant les idées innovantes dans les domaines de l'ingénierie et du management}

TRIZ: favoriser l'inventivité et résoudre des problèmes techniques

Le modèle quantique, à l'instar du théorème de Thalès, introduit une innovation scientifique grâce à des opérations de recadrage, ainsi que nous l'avons montré. Cependant, il le fait de manière implicite. Or, il nous paraît plus fructueux d'étudier des méthodes explicitement et résolument tournées vers I'innovation. Parmi les chercheurs ayant élaboré des méthodes destinées à stimuler la créativité dans ce domaine, deux figures se distinguent par le caractère pionnier de leur approche, mais aussi par l'efficacité de leurs outils: Genrich Altshuller et Edward de Bono. Le premier a mis en place une méthode baptisée TRIZ, qui contribue actuellement à bouleverser le secteur de I'industrie ${ }^{3}$. Le second est l'auteur de la pensée latérale et des "Six chapeaux», mondialement connus par les «gourous» du management puisqu'il développa notamment la méthode du brainstorming.

Né en 1926 dans l'ex-URSS, Genrich Altshuller se fait remarquer très jeune par les services d'expertise en brevet d'invention de la marine russe. Il devient rapidement leur expert principal, mais son désir d'autonomie lui fait quitter des fonctions jugées trop restrictives. Il s'attelle alors à l'analyse des mécanismes inventifs à l'œuvre chez les grands esprits créatifs de l'Histoire - tel Léonard de Vinci - et ambitionne d'élaborer une théorie facilitant et systématisant «le processus de génération d'idées dans le cadre des phases de recherche de concepts et de résolution de problèmes» (Cavallucci, 1999; chap. 1), grâce à la construction d'une matrice. En 1946 apparaît pour la première fois TRIZ, qui répond à ces objectifs. Cet acronyme cyrillique est traduit, en français, par "Théorie de Résolution des Problèmes d'Innovation ». À partir de 1951, son auteur commencera à fonder des universités TRIZ dans son pays natal, afin de diffuser sa théorie, soutenu par un groupe de chercheurs convaincus de la pertinence de sa méthode. À partir de 1956, elle s'enrichit d'un ARIZ (Algorithme de Résolution des Problèmes d'Innovation). Malgré son originalité, TRIZ ne passera les frontières qu'après la chute du mur de Berlin. Elle commencera à faire fureur aux États-Unis dès 1991, avant de conquérir une partie de l'Europe ${ }^{4}$.

Genrich Altshuller part du principe que la plupart des problèmes d'innovation résident dans des contradictions supposées insurmontables, notamment à cause de ce qu'il appelle «l'inertie psychologique», que l'on peut définir comme un formatage de la pensée qui amène inconsciemment le chercheur à entrevoir toute expérience à travers une structure mentale rigide et préétablie (et qui, par conséquent, le rend incapable de dépasser les apories, en développant des voies alternatives). II recense trois types de contradictions: la contradiction d'état, la contradiction technique et la contradiction physique. Cette dernière nous intéresse plus particulièrement, car « elle oppose directement deux requêtes (ou paramètres) formulées par un seul et même système [et met] en évidence le caractère impossible de la situation", ainsi que le note Denis Cavallucci (1999: chap. 2.2) dans un article consacré à TRIZ. Les spécialistes en sciences de l'information et de la communication établiront aisément un parallèle avec le concept de «double contrainte» («double bind») mis en exergue par Gregory Bateson au cours des études qu'il mena sur des familles de schizophrènes, et désignant la position intenable d'un individu qui se trouve soumis à deux messages contradictoires (ou deux « injonctions paradoxales»). L'ingénieur qui s'efforce de répondre à des exigences techniques incompatibles est dans une situation assez similaire.

Mais les similitudes ne s'arrêtent pas là. Au-delà de l'identification de problèmes voisins, les solutions proposées tant par Gregory Bateson, puis par Paul Watzlawick dans le domaine thérapeutique, que par Genrich Altshuller dans le domaine de l'innovation technique se ressemblent à bien des égards. En effet, à l'instar des chercheurs de Palo Alto, le scientifique russe s'achemine vers une résolution systémique du problème. Il préconise ainsi de ne pas se limiter à un seul champ d'investigation, mais d'envisager la difficulté dans sa totalité afin d'en avoir une vision globale. Ainsi un problème de nature mécanique, apparemment rédhibitoire, pourra-t-il être résolu grâce à la chimie ou l'électronique, par exemple, c'est-à-dire par déplacement du cadre de réflexion. Ces remarques ne sont pas sans rappeler les principes de la thérapie familiale émis par Gregory Bateson, principes selon lesquels la maladie d'un être humain est comprise comme un dysfonctionnement du 
groupe auquel il appartient plutôt que comme un problème purement individuel.

Enfin, de la même manière que les chercheurs du MRI utilisaient la communication pour dénouer une situation familiale pathogène et guérir un patient atteint de schizophrénie, Genrich Altshuller utilise couramment le principe de reformulation afin de briser l'inertie psychologique et lever les difficultés faisant obstacle à l'esprit de créativité et aux solutions innovantes. Denis Cavallucci souligne à ce propos qu'il s'agit de

[...] déplacer, par la formulation, l'angle de vision de la pensée en la plaçant dans des situations extrêmes. Ceci afin de donner une vision différente du problème parfois plus propice pour y entrevoir la solution. (Ibid. : chap. 4.3)

Donnons un exemple concret de déplacement terminologique, entraînant un recadrage et permettant l'amélioration d'un système technique: soit un système d'impression couleur doté d'un rouleau, ayant pour fonction, lors de sa mise en rotation en opposition au défilement du film photoélectrique, d'expulser par répulsion les surplus de liquide. Dans le but d'accroître les performances de l'appareil, les ingénieurs du projet ont mis en évidence que l'augmentation du diamètre du rouleau maximiserait l'opération d'expulsion. Mais l'augmentation du diamètre soulève un problème, car il occupe démesurément l'espace et nuit au fonctionnement global du système, qui devient plus encombrant. Si l'on formule le problème qu'il convient de résoudre par «remplacer le rouleau», on invite inconsciemment l'ingénieur à chercher une pièce de remplacement cylindrique. En revanche, une formulation du type «remplacer la pièce par un objet qui doit évacuer les surplus d'encre», on brise une barrière psychologique et l'ingénieur sera plus enclin à chercher des solutions alternatives vraiment innovantes: une courroie, par exemple, lève la contradiction initiale, puisqu'elle permet une mise en rotation et encombre moins l'espace. D'où cette conclusion: «le premier réflexe dans la formulation des phrases de mise en situation est d'identifier ces mots porteurs d'inertie et de les remplacer par d'autres, plus neutres» (Ibid. : chap. 2.3).

Genrich Altshuller démontre donc comment la formulation d'une problématique s'avère essentielle, le choix des éléments de langage influençant la façon dont I'homme conçoit un problème et sa résolution. Un simple déplacement terminologique (l'utilisation de termes dénués de connotation et donc de détermination préalable) peut rendre possible l'innovation. Une autre méthode de recadrage implicite nous est livrée par
Altshuller à travers la "théorie des hommes miniatures", qui est comme un écho de la méthode empathique de l'ingénieur américain William Gordon. Celle-ci consiste, pour l'inventeur, à imaginer qu'il est un personnage minuscule à l'intérieur du système dont il tente de résoudre un problème technique. Ce changement d'échelle du cadre de référence est censé favoriser une appréhension différente des éléments en présence, une proximité avec les diverses parties de l'appareil. Cette théorie des hommes miniatures démontre que si la créativité, pour le concepteur de TRIZ, n'est pas tant un art qu'une science exacte (Altshuller, 1988), elle implique néanmoins un équilibre entre analyse et imagination, technique et psychologie.

En conclusion, I'on peut dire que l'obstacle technique tel qu'Altshuller l'envisage, et qui aboutit à une incapacité à aller jusqu'au bout d'une découverte innovante, pourrait être comparé au symptôme étudié par Paul Watzlawick dans le cadre des maladies mentales, rendant impossible la guérison du patient. En effet, l'obstacle technique, comme le symptôme, est dépendant d'un contexte figé et inadéquat, qui empêche l'individu de penser ou de se comporter autrement. Pour cette raison même, l'opération de recadrage, même si elle n'est pas nommée comme telle, est omniprésente dans la réflexion d'Altshuller. Que ce soit à travers une approche systémique, une reformulation par déplacement terminologique ou un changement d'échelle, elle est la méthode privilégiée par l'un des plus grands spécialistes de l'innovation parce qu'elle rend possible une lecture originale de dysfonctionnements en tous genres.

La "pensée latérale»,

ou comment faire naître les idées innovantes

Dans son ouvrage sur l'intelligence économique (2004), François Jakobiak rappelle que les innovations sont de deux sortes: matérielles et immatérielles. La première catégorie comprend essentiellement les innovations de produits et de procédés techniques, auxquelles Genrich Altshuller a consacré une part importante de ses études. La seconde concerne les innovations de services et les innovations organisationnelles. S'il n'ignore pas les innovations techniques, Edward de Bono s'est essentiellement intéressé au second type. Bien que leurs objets d'étude soient quelque peu différents, les théories de Genrich Altshuller et de Edward de Bono possèdent des approches similaires, ainsi que nous allons le démontrer.

Les recherches de Edward de Bono se nouent autour de la notion de «pensée latérale» («lateral thinking»), qui est d'ailleurs devenue le titre de l'un de ses ouvrages (1972). L'auteur définit la 
pensée latérale comme « une manière différente et plus créatrice d'utiliser l'esprit ». La présentation qu'il en fait n'est d'ailleurs pas sans rappeler cette «pensée oblique» que les Grecs avaient incarnée en Métis, avant de la sacrifier à un type d'intelligence plus rationnelle (Vernant et Detienne, 1974)... Edward de Bono illustre ses propos en évoquant les mathématiques modernes, lesquelles, "abandonnant l'ancienne conception rigide», très proche du phénomène $d^{\prime}$ 'inertie psychologique décrit par Altshuller, encouragent une plus grande souplesse puisqu'elles invitent «l'élève à envisager un même problème sous plusieurs angles différents et à se rendre compte qu'il peut exister diverses manières d'arriver à une conclusion exacte» (De Bono, 1972 : 11-12). Ce discours corrobore implicitement la théorie constructiviste des chercheurs de Palo Alto, et plus précisément les deux niveaux de réalité mis en évidence par Paul Watzlawick. Les divers angles d'approche dont parle Edward de Bono, en effet, se rapportent à une réalité constituée de valeurs et de représentations symboliques, tandis que la conclusion exacte à laquelle il se réfère par ailleurs se rapporte à la réalité des propriétés physiques des objets.

Cette pensée latérale, il l'oppose à la "pensée verticale». Prégnante dans la culture occidentale, celle-ci procède d'une démarche logique et fut initialement formalisée par la philosophie aristotélicienne. Or, si cette faculté de rationalisation se révèle décisive dès lors qu'il s'agit de procéder à une analyse rigoureuse et objective, elle est en revanche globalement incapable d'innover. La pensée verticale, en effet, est productrice de rigidité et d'inertie, selon l'auteur, car elle est attachée à un carcan intellectuel. Des idées dominantes, polarisantes (que l'on pourrait appeler des préjugés, ou plus simplement des structures mentales conditionnantes), influencent le mode de raisonnement et, partant, le choix de conception ou de solution proposé pour faire évoluer un procédé, un service ou encore une organisation. À l'inverse, la pensée latérale fait naître des idées créatives, inattendues, qui permettent de sortir d'un dilemme, d'un problème lorsque celui-ci semble n'offrir qu'un choix binaire, propre au principe de contradiction posé par la logique. Devant des options qui paraissent limitées et donc également insatisfaisantes, la pensée latérale peut trouver des voies alternatives. Polymorphe, elle a pour spécificité de s'adapter à des situations inédites.

Edward de Bono semble avoir été directement influencé par les théories prégnantes dans les SIC, notamment celles de Norbert Wiener et de l'École de Palo Alto, et ce, même s'il ne revendique pas explicitement une telle filiation de pensée. En effet, lorsqu'il définit la pensée latérale, il se réfère aux notions de «feedback positif » et de «feedback négatif», puis mentionne le « jeu complexe de leur interaction au sein du système » (ibid. : 11). Autre parallèle surprenant, de Bono compare l'inventeur qui utilise la pensée latérale "aux associations fortuites du schizophrène", précisant toutefois que si les deux échappent «au point de vue dominant», l'un contrôle ce processus, mais I'autre ne le peut pas (ibid. : 29-30). Dernière similitude, il utilise des recadrages grâce au langage, procédé courant chez Paul Watzlawick mais également chez Genrich Altshuller, ainsi que nous I'avons vu. Edward de Bono déclare ainsi : «la rigidité des mots est associée à la rigidité des classifications. Répétons que la rigidité des classifications conduit à la rigidité des points de vue». Puis il poursuit: «les mots et les noms disponibles déterminent la façon dont une situation donnée peut être envisagée » (ibid.: 143-144). Et de donner quelques exemples proches de celui que nous avons étudié avec Altshuller concernant un système d'impression couleur où le terme "rouleau » faisait obstacle à une solution innovante, et avait été avantageusement remplacé par le mot «objet». Parmi ces exemples, on compte celui du système hydraulique défectueux d'un avion de la Seconde Guerre mondiale, pouvant entraîner un écrasement. Dépassant le sens restrictif des termes "système hydraulique», la pensée latérale peut ainsi pallier les fuites en remplissant le réservoir avec l'urine des membres de l'équipage... Pour éviter le déterminisme mental dont sont porteurs les signifiants - et que les publicistes et les sémiologues, spécialistes de l'analyse de la connotation des messages, connaissent bien-Edward de Bono exhorte à réfléchir à l'aide d'images, de manière très visuelle, en dehors de tout système linguistique.

L'opération de recadrage, enfin, est la clé de voûte de sa fameuse méthode des "Six chapeaux» ("Six Thinking Hat»), qui a inspiré de nombreux managers et a contribué à la notoriété de brainstorming (initialement conçu par le publicitaire américain Alex Osborn) dans les entreprises, vers la fin des années 1980. Conçue comme un jeu de rôles, cette méthode consiste à endosser six chapeaux de couleurs différentes, chaque chapeau étant associé à des comportements et des modes de réflexions spécifiques: le blanc correspond à une présentation neutre, objective, des faits et informations disponibles; le rouge, aux sentiments, à des réactions émotionnelles dépourvues de justification rationnelle; le noir, au jugement critique, froidement logique; le vert, à la pensée créatrice, à des hypothèses originales, etc. Chaque cas présenté est ainsi abordé sous des aspects variés. L'objectif de cet exercice est évidemment de faire éclater les limitations habituelles en obligeant un individu 
à embrasser des perspectives multiples, qui sont comme les nombreuses facettes d'un prisme appelé « réalité ». L'intérêt des travaux de Edward de Bono réside également dans le fait qu'il introduit une dimension collective de l'innovation, qui se trouvait absente dans les écrits d'Altshuller. II rejoint donc, dans une certaine mesure, les travaux de la sociologie de la traduction et de la théorie de l'acteur en réseau (aussi connue sous l'abréviation ANT, soit Actor-Network Theory) développées par Madeleine Akrich, Michel Callon et Bruno Latour (2006). Or, ces trois chercheurs de l'École des mines de Paris ont eux aussi emprunté le concept central de «traduction » à Michel Serres, et plus précisément aux réflexions que ce philosophe a menées dans le domaine des SIC (1974).

Nous nous sommes efforcée de démontrer l'importance que revêt la technique de recadrage, développée par le Collège invisible, dans le processus d'innovation, et plus largement dans tout processus de changement. Dans cette perspective, nous avons également montré, en nous appuyant sur les méthodes innovantes de Genrich Altshuller et de Edward de Bono, que des analyses de langage permettent de dépasser un certain déterminisme mental et favorisent ainsi la créativité.

L'apport des SIC, et notamment du courant constructiviste, se révèle donc essentiel dans le domaine de l'ingénierie comme dans celui du management par ses différentes techniques de remodélisation. Cependant, I'utilisation qui est faite de ces outils communicationnels par des chercheurs innovants est bien souvent inconsciente. Une formalisation et un usage systématique de ces techniques pourraient en maximiser les effets. Nous préconisons, par conséquent, d'intégrer le procédé de recadrage par déplacement terminologique, dans les méthodes destinées à favoriser l'innovation, comme une étape préalable indispensable. Cela devrait augmenter l'efficacité des recherches tournées vers l'innovation et permettre en outre une plus grande prise de conscience de la richesse que crée l'interdisciplinarité.

\footnotetext{
NOTES

1. SIC: Sciences de l'information et de la communication.

2. L'expression «Collège invisible», que l'on doit à Yves Winkin (1981), désigne un courant de pensée qui se forma à Palo Alto, en Californie, dans les années 1950-1960, grâce au réseau informel de chercheurs venant d'horizons différents: Paul Watzlawick, Gregory Bateson, Edward T. Hall, Ray Birdwhistell, Erving Goffman, notamment, travaillèrent sur le thème de la communication interpersonnelle et des dynamiques sociales et s'efforcèrent de dépasser une conception télégraphique de la communication.
}

3. Des logiciels s'appuyant sur TRIZ ont d'ailleurs vu le jour, et le Massachusetts Institute of Technology a récemment mené des études sur cette théorie et prouvé qu'un chercheur se montrait $70 \%$ plus inventif avec I'aide de TRIZ.

4. Parmi les traductions françaises de ses ouvrages, voir 40 Principes d'innovation. TRIZ pour toutes applications (2004) et Et soudain apparut I'inventeur. Les idées de TRIZ (2002).

\section{RÉFÉRENCES BIBLIOGRAPHIQUES}

AKRICH, M., M. CALLON et B. LATOUR [2006]: Sociologie de la traduction. Textes fondateurs, Paris, Presses de l'École des mines, coll. "Sciences sociales».

Altshuller, G. [1988] : Creativity as an Exact Science, New York, Gordon \& Breach ;

[2002] : Et soudain apparut l'inventeur. Les idées de TRIZ, Paris, Avraam Seredinski :

[2004] : 40 Principes d'innovation. TRIZ pour toutes applications, Paris, Avraam Seredinski.

BATESON, G. [1971] : La Cérémonie du naven, Paris, Minuit; [1980]: Vers une écologie de l'esprit, 2 tomes, Paris, Seuil.

BRETON, A. [1985] : Manifestes du surréalisme, Paris, Gallimard, coll. «Folio essais».

CALlon, M. [1988] : La Science et ses réseaux. Genèse et circulation des faits scientifiques, Paris, La Découverte.

CAVALLUCCI, D. [1999] : "TRIZ: I'approche altshullerienne de la créativité », Techniques de l'ingénieur. En ligne: http://www.techniquesingenieur.fr/base-documentaire/genie-industriel-th6/conception-etproduction-ti083/triz-l-approche-altshullerienne-de-la-creativite-a5211/ (page consultée le 24 février 2011).

De Bono, E. [1972] : La Pensée latérale, Paris, Stock; [1987] : Six chapeaux pour penser, Paris, Interéditions.

Goffman, E. [1974] : Les Cadres de l'expérience, Paris, Minuit.

JАКОВІак, F. [2004] : L'Intelligence économique. La comprendre, l'implanter, l'utiliser, Paris, Éd. d'organisation.

KLEIN, É. [1997] : La Physique quantique, Paris, Flammarion. Koestler, A. [1965] : Le Cri d'Archimède, Paris, Calmann-Lévy. KUHN, T. [1999] : La Structure des révolutions scientifiques, Paris, Flammarion.

MuCCHIELLI, A. [2004] : Étude des communications. Approche par les processus, Paris, Armand Colin.

PLATON [1993]: La République, Paris, Flammarion.

Russo, F., C. Rea et L. SAlamanCA-AviLA [2004] : "L'École de LouvainLa-Neuve est-elle constructiviste? », dans A. Mucchielli (dir.), La Place du constructivisme pour l'étude des communications. Actes du colloque (avril 2003), Béziers, Publications Montpellier 3, 163-183.

SERres, M. [1974] : Hermès III. La Traduction, Paris, Minuit.

SIMAY, P. [2008] : "Une autre ville pour une autre vie. Henri Lefebvre et les situationnistes », Métropoles, no 4 . En ligne: http://metropoles. revues.org/2902\#text (page consultée le 24 février 2011).

Vernant, J.-P. et M. Detienne [1974] : Les Ruses de l'intelligence. La Métis des Grecs, Paris, Flammarion.

WatzlaWick, P. [1977] : How Real is Real? Confusion, Disinformation, Communication, New York, Vintage;

- [1984] : Faites vous-même votre malheur, Paris, Seuil ;

[1991] : Les Cheveux du baron de Münchhausen, Paris, Seuil.

WATZLAWICK, P., J. WEAKLAND et R. FISCH [1975] : Changements : paradoxes et psychothérapie, Paris, Seuil.

WINKIN, Y. [1981] : La Nouvelle Communication, Paris, Seuil. 